though in the case of Drosophila drag effects dominate-it resembles an aircraft working at the limit of its ability with almost nothing to spare for manoeuvres.

The trouble about an oscillating propellor, as provided by wings beating at a frequency of 35 per second in the humming-bird or 250 per second in the fruit fly, is the enormous amount of energy involved in the stopping and starting of the wings twice in each cycle. In insects, as was shown a good many years ago by Weis-Fogh, not more than 15 per cent of this energy is degenerated into heat ; at least 85 per cent is stored as elastic energy and released as kinetic energy during the reversed stroke. This elastic storage is effected by the solid skeletal cuticle of the thoracic wall, by the remarkable elastomer resilin in the wing hinges and wing stops, and by an elastic component in the myofibrils themselves.

It seems that the humming-bird is unable to make economies of this kind: elastic components in the opposing muscles are poorly developed; the osseous internal skeleton lacks suitable elasticity; and although elastin-the common elastomer in vertebrates-can serve this purpose during running and walking, its damping properties rise so steeply at high frequencies of oscillation that it cannot operate effectively in the beating wings. It looks as though the vertebrates selected the wrong elastomer at an early stage of evolution-before flight was envisaged.

\section{SOLAR SYSTEM}

\section{Too Many Cooks}

from a Correspondent

THE origin of the solar system is one of the oldest unsolved problems in natural philosophy, and at a discussion meeting organized jointly by the Royal Society and the Royal Astronomical Society on March 27 astronomers and space scientists once again learnt that there are as many theories on the subject as there are researchers in the field. Dr D. Ter Haar (University of Oxford) went so far as to suggest that a solution is no nearer now than it was in René Descartes's time 340 years ago.

Theories proliferate because of the scarcity of facts. At present the chief facts which have to be explained are: (1) the regularity of the system-the planets move in prograde, near circular orbits and have isochronous spins about axes which are largely perpendicular to the ecliptic; (2) the satellite distribution around a planet is similar to the planetary distribution around the Sun; (3) the division into an inner group of small, dense, satellite-free planets and an outer group of large, low density, multisatellite planets; (4) the peculiar regularities in the planetary and satellite spacings, summed up by the Titius-Bode law; and finally (5) the distribution of angular momentum and mass between the Sun and the planets.

Most of the theories which have been propounded had the planets condensing out of a nebula of gas and dust surrounding the Sun. Where the nebula came from and why, and whether or not it postdated the Sun, were questions left unanswered. Dr Ter Haar showed that turbulent eddies could lead to condensations with different compositions and densities, and these differences are caused by the decrease in nebula temperature as the planet moves away from the Sun. A serious problem arises here because the accretion of a planet would take about $10^{8} \mathrm{yr}$ and this is a factor $10^{5}$ greater than the eddy lifetime. Dr Ter Haar also stressed the important similarities between planet and satellite formation.

Discussing the angular momentum deficiency of the Sun, Professor G. Arrhenius (Scripps Institution of Oceanography) concluded that magneto-hydrodynamic breaking must have occurred and that this ionized the nebula. $\mathrm{He}$ discussed recent experiments on the condensation of magnesium silicate and glasses from cold (a few hundred degrees Kelvin) rarefied plasmas, and concluded that meteorites have been formed by direct condensation and not by secondary processes in planetary mantles.

Professor N. M. Woolfson (University of York) drew the audience back to the stellar approach theory of Jeans, but with a difference, because he suggested that the planet-forming material was drawn out of the passing star and not from the Sun. Dr D. G. King-Hele (Royal Aircraft Establishment, Farnborough) then delved into the realms of mysticism and numerology as he amusingly told of the empathic mythopia of the poetical descriptions of the birth of the solar system and also the numerical juggling which produced the Pythagorean harmony of the spheres and the Keplerian solution which used a nest of regular polyhedra. By extending the Titius-Bode law he showed that there are very significant relationships between the parameters of the different planetary orbits. Professor W. H. McCrea (University of Sussex) discussed the protoplanet theory in which, for example, Earth, Mars and the Moon were all formed from one large body which became unstable and broke up.

Professor S. K. Runcorn (University of Newcastle upon Tyne) summed up by reminding the audience that a sure way forward was to look for more

\title{
Lysogeny at Random in Escherichia coli
}

AT the root of the lysogeny of Escherichia coli cells by the bacteriophage $\lambda$ lies a recombination event between a part of the circular phage chromosome and a specific site in the host cell chromosome. In other words, unique phage and cell DNA sequences recognize each other and the $\lambda$ DNA is integrated at the recognition or attachment site. P2, another lysogenic phage, is somewhat more catholic than phage $\lambda$, for it can integrate at more than one site, but still a limited number of sites, in the host chromosome. Phage Mu-1 is remarkable, however, for its extreme catholicity; it seems to be able to integrate at virtually any site in the $E$. coli $\mathrm{K} 12$ genome. Does this mean that a base sequence specifically recognized by the Mu-1 DNA is repeated at countless sites in the bacterial chromosome, or does the Mu-1 integration machinery integrate the Mu-1 DNA at any site regardless of the host base sequence? Experiments reported by Bukhari and Zipser in next Wednesday's Nature New Biology (April 26) clearly indicate that the latter alternative is indeed the case; the base sequence of the host DNA seems to be irrelevant to integration of Mu-1 DNA.

Bukhari and Zipser have established this by analysing the pattern of integration of Mu-1 DNA within the $z$ gene of the lactose operon, with which they are very familiar. In essence, by deletion mapping, they have shown that the phage DNA can integrate at at least seventy-six sites in this one gene, which is tantamount to proving that integration can occur at random in this gene and presumably therefore in other $E$. coli genes.

Irrespective therefore of whether or not one special region of the Mu-1 DNA is involved in integration, a point which Bukhari and Zipser are pursuing, it is obvious that specific host sequences are not required, a finding which should greatly stimulate efforts to isolate and characterize the $\mathrm{Mu}-1$ integration enzymes.

Bukhari and Zipser have also observed that recombination between two strains carrying Mu-1 DNA integrated at close but not identical sites in the $z$ gene is a very rare event. It also seems that the presence of the $\mathrm{Mu}-1$ DNA somehow excludes recombination in adjacent regions of the $z$ gene, and this is not the result of deletion of host DNA caused by integration of the phage DNA. It seems more likely, according to Bukhari and Zipser, that the pairing of the prophages themselves on mating throws out of alignment adjacent host DNAs and therefore prevents crossovers of host DNA. 